\title{
Associação entre sinais de sofrimento psíquico até dezoito meses e rebaixamento da qualidade de vida aos seis anos de idade ${ }^{1}$
}

\author{
Angela Di Paolo Mota ${ }^{a *}$ \\ Rogério Lerner ${ }^{\mathrm{a}}$ \\ Ana Maria de Ulhôa Escobarb \\ Maria Cristina Machado Kupfer ${ }^{\mathrm{a}}$ \\ Francisco Marcelo Monteiro da Rochac \\ Luiz Silva dos Santos ${ }^{a}$ \\ aUniversidade de São Paulo, Instituto de Psicologia. São Paulo, SP, Brasil \\ 'Universidade de São Paulo, Faculdade de Medicina. São Paulo, SP, Brasil \\ 'Universidade Federal de São Paulo (Unifesp), São Paulo, SP, Brasil
}

\begin{abstract}
Resumo: A pedido do Ministério da Saúde foi desenvolvido e validado um protocolo de Indicadores clínicos de Risco para o Desenvolvimento Infantil (IRDI) para detectar riscos para transtornos psíquicos de desenvolvimento em bebês de 0 a 18 meses. Este trabalho teve como objetivo verificar a validade preditiva do IRDI, a partir de sua relação com dois instrumentos: o Autoquestionnaire Qualité de Vie Enfant Imagé (AUQUEI) e o Child Health Questionnaire (CHQ), ambos já validados no Brasil e aplicados aos seis anos de vida. A amostra foi composta por 46 crianças de três centros de saúde de São Paulo. Os resultados indicaram que o IRDI não se mostrou sensível à avaliação de qualidade de vida obtida com o uso do AUQUEI. No entanto, a associação estatisticamente significante encontrada entre resultados obtidos no IRDI e no CHQ mostrou maior capacidade do IRDI para predizer qualidade de vida relacionada ao índice psicossocial do que ao índice físico do mesmo instrumento.
\end{abstract}

Palavras-chave: qualidade de vida, desenvolvimento infantil, psicanálise.

\section{Introdução}

Um grupo de pesquisadores brasileiros ${ }^{2}$ desenvolveu e validou no período de 2000 a 2008, com financiamento da FAPESP, do Ministério da Saúde e do CNPq, um protocolo

1 Agradecemos à Universidade de São Paulo, pelo auxílio necessário à licença de uso do Child Health Questionnaire. À Fundação de Amparo à Pesquisa do Estado de São Paulo, pela concessão da bolsa de estudos e pelo auxílio financeiro necessários à realização desta pesquisa. À Maria Eugênia Pesaro, por permitir o acesso ao banco de dados da Pesquisa IRDI. Ao Bruno Molina Turra, pela revisão e tradução do texto para o inglês. Aos coordenadores e profissionais dos centros de saúde que viabilizaram a pesquisa de campo deste estudo: Hospital Universitário da Universidade de São Paulo, Centro de Saúde Escola Samuel Barnsley Pessoa e Programa Einstein na Comunidade de Paraisópolis. A todos os participantes da pesquisa, pela atenção e colaboração para que o estudo acontecesse.

2 Um grupo nacional de pesquisa (denominado GNP), sob coordenação geral da profa. dra. Maria Cristina Machado Kupfer, foi estabelecido para o desenvolvimento desta pesquisa. O grupo foi constituído pela profa dra. Leda M. Fischer Bernardino, da PUC de Curitiba, Paula Rocha e Elizabeth Cavalcante, do CPPL de Recife, Domingos Paulo Infante, Lina G. Martins de Oliveira e M. Cecília Casagrande, de São Paulo, Daniele Wanderley, de Salvador, profa. Lea M. Sales, da Universidade Federal do Pará, profa. Regina M. R. Stellin, da UNIFOR de Fortaleza, Flávia Dutra, de Brasília, prof. dr. Otavio Souza, do Rio de Janeiro, Silvia Molina, de Porto Alegre, com coordenação técnica de Maria Eugênia Pesaro, coordenação científica do dr. Alfredo Jerusalinsky e coordenação científica nacional da profa. dra. Maria Cristina Machado Kupfer.

* Autora correspondente: angeladipaolo@usp.br de Indicadores clínicos de Risco para o Desenvolvimento Infantil (IRDI) a fim de detectar riscos psíquicos e problemas de desenvolvimento em bebês de 0 a 18 meses.

Ao final de 2008, 280 crianças completaram o estudo, também chamado de pesquisa IRDI, sendo acompanhadas aos três anos, com dois outros instrumentos: o Strengths and Difficulties Questionnaire (SDQ), desenvolvido por Robert Goodman em 1997, e a Avaliação Psicanalítica aos 3 anos (AP3) para fins de realização do desfecho clínico da pesquisa (Kupfer et al., 2008, 2009, 2010; Lerner \& Kupfer, 2008).

Tal protocolo é composto por 31 indicadores, como se pode observar na Figura 1. Os indicadores são aplicados de acordo com a seguinte faixa etária correspondente: de 0 a 4 meses incompletos, de 4 a 8 meses incompletos, de 8 a 12 meses incompletos e de 12 a 18 meses.

Considerou-se que problemas de desenvolvimento sinalizam a presença de dificuldades subjetivas que afetam ou incidem no desenvolvimento da criança sem questionar a instalação do sujeito psíquico. Risco psíquico, por sua vez, seria uma categoria que compreende as dificuldades de desenvolvimento sinalizadoras de entraves no processo de constituição subjetiva, apontando problemas mais estruturais, um risco de evolução em direção às psicopatologias graves da infância, tais como os chamados distúrbios globais do desenvolvimento. Os resultados apontaram a 


\begin{tabular}{|c|c|}
\hline Idade em meses: & Indicadores: \\
\hline 0 a 4 meses incompletos: & $\begin{array}{l}\text { 1. Quando a criança chora ou grita, a mãe sabe o que ela quer. } \\
\text { 2. A mãe fala com a criança num estilo particularmente dirigido a ela (manhês). } \\
\text { 3. A criança reage ao manhês. } \\
\text { 4. A mãe propõe algo à criança e aguarda a sua reação. } \\
\text { 5. Há trocas de olhares entre a criança e a mãe. }\end{array}$ \\
\hline 4 a 8 meses incompletos: & $\begin{array}{l}\text { 6. A criança começa a diferenciar o dia da noite. } \\
\text { 7. A criança utiliza sinais diferentes para expressar suas diferentes necessidades. } \\
\text { 8. A criança solicita a mãe e faz um intervalo para aguardar sua resposta. } \\
\text { 9. A mãe fala com a criança dirigindo-lhe pequenas frases. } \\
\text { 10. A criança reage (sorri, vocaliza) quando a mãe ou outra pessoa está se dirigindo a ela. } \\
\text { 11. A criança procura ativamente o olhar da mãe. } \\
\text { 12. A mãe dá suporte às iniciativas da criança sem poupar-lhe o esforço. } \\
\text { 13. A criança pede a ajuda de outra pessoa sem ficar passiva. }\end{array}$ \\
\hline 8 a 12 meses incompletos: & $\begin{array}{l}\text { 14. A mãe percebe que alguns pedidos da criança podem ser uma forma de chamar a sua } \\
\text { atenção. } \\
\text { 15. Durante os cuidados corporais, a criança busca ativamente jogos e brincadeiras } \\
\text { amorosas com a mãe. } \\
\text { 16. A criança demonstra gostar ou não de alguma coisa. } \\
\text { 17. Mãe e criança compartilham uma linguagem particular. } \\
\text { 18. A criança estranha pessoas desconhecidas para ela. } \\
\text { 19. A criança possui objetos prediletos. } \\
\text { 20. A criança faz gracinhas. } \\
\text { 21. A criança busca o olhar de aprovação do adulto. } \\
\text { 22. A criança aceita alimentação semissólida, sólida e variada. }\end{array}$ \\
\hline De 12 a 18 meses: & $\begin{array}{l}\text { 23. A mãe alterna momentos de dedicação à criança com outros interesses. } \\
\text { 24. A criança suporta bem as breves ausências da mãe e reage às ausências prolongadas. } \\
\text { 25. A mãe oferece brinquedos como alternativas para o interesse da criança pelo corpo } \\
\text { materno. } \\
\text { 26. A mãe já não se sente mais obrigada a satisfazer tudo que a criança pede. } \\
\text { 27. A criança olha com curiosidade para o que interessa à mãe. } \\
\text { 28. A criança gosta de brincar com objetos usados pela mãe e pelo pai. } \\
\text { 29. A mãe começa a pedir à criança que nomeie o que deseja, não se contentando apenas } \\
\text { com gestos. } \\
\text { 30. Os pais colocam pequenas regras de comportamento para a criança. } \\
\text { 31. A criança diferencia objetos maternos, paternos e próprios. }\end{array}$ \\
\hline
\end{tabular}

Figura 1. Indicadores Clínicos de Risco para o Desenvolvimento Infantil (IRDI)

Fonte: Kupfer et al. (2010)

capacidade do IRDI para predizer problemas de desenvolvimento e destacaram ainda 15 indicadores com capacidade de predizer risco psíquico para a constituição subjetiva (Kupfer et al., 2008, 2009, 2010; Lerner \& Kupfer, 2008).

Acerca dos resultados finais da pesquisa, Kupfer et al. (2008, p. 225) destacaram quatro indicadores sensíveis à predição de risco psíquico, medido pelo risco relativo, estatisticamente significante: os indicadores número $7(\mathrm{RR}=$ 3,46; IC95\% 1,19 - 10,07), número $18(\mathrm{RR}=2,93$; IC95\% 1,49 - 5,73), número 22 ( $R R=3,75$; IC95\% 1,37 - 10,28) e número $30(\mathrm{RR}=4,19$; IC95\% 1,74-10,06).

Tais indicadores foram erigidos a partir de eixos teóricos extraídos da teoria psicanalítica, que são: suposição do sujeito, estabelecimento da demanda, alternância entre presença e ausência e instalação da função paterna, todos indicativos da instalação do sujeito psíquico. As bases psicanalíticas do IRDI foram discutidas em Kupfer et al. $(2009,2010)$ e Pesaro (2010).

Uma primeira utilização do IRDI já foi realizada: alguns indicadores serviram de base para a construção dos itens "Desenvolvendo-se com afeto" da Caderneta de Saúde da Criança, adotada pela Área de Saúde da Criança (responsável pelo financiamento da pesquisa mencionada, em associação com a FAPESP) do Ministério da Saúde (Brasil, 2007).

A pesquisa com o IRDI gerou um banco de dados que tem contribuído para ampla produção bibliográfica (incluindo iniciações científicas, dissertações, teses, livros, trabalhos publicados e apresentações em eventos). Entre as produções recentes, pode-se destacar o trabalho de Pesaro (2010) que, em tese de doutorado, analisa a aplicação de métodos distintos em pesquisas, voltando-se especialmente para o lugar da psicanálise na pesquisa com os indicadores clínicos. 
Em dissertação de mestrado, Di Paolo (2010) teve como objetivo geral constituir um estudo exploratório de associação entre resultados obtidos no IRDI e na AP3 e resultados referentes à qualidade de vida e à condição sintomática da criança aos seis anos. Os resultados apontaram para uma associação significativa entre os indicadores clínicos e o índice psicossocial do CHQ-PF50 (um dos instrumentos utilizados para avaliar qualidade de vida). Concluiu-se que o IRDI é capaz de detectar uma tendência de ocorrência de problemas expressa nos 18 primeiros meses de vida que se estende ao longo do desenvolvimento da criança pelo menos até o sexto ano, levando ao impacto na sua qualidade de vida a partir da perspectiva de seus pais.

Lerner (2011) pesquisou a capacidade discriminativa do IRDI entre autismo, retardo mental e normalidade. Os seguintes itens do IRDI mostraram capacidade discriminativa estatisticamente significativa: IRDI 5 (há troca de olhares entre a criança e a mãe), IRDI 10 (a criança reage - sorri, vocaliza - quando a mãe ou outra pessoa está se dirigindo a ela), IRDI 16 (a criança demonstra gostar ou não de alguma coisa), IRDI 29 (a mãe começa a pedir à criança que nomeie o que deseja, não se contentando apenas com gestos) e IRDI 30 (os pais colocam pequenas regras de comportamento para a criança). Concluiu-se que 0 IRDI tem poder de discriminar autismo de retardo mental e normalidade, justificando que se façam pesquisas acerca de sua utilização na deteç̧ão de quadros de transtorno do espectro de autismo, incorporando itens dos outros instrumentos que também tiveram capacidade discriminativa.

O presente estudo incidiu sobre a verificação do grau de associação entre o IRDI e dois instrumentos que avaliam qualidade de vida da criança aos seis anos, a partir de diferentes concepções, o AUQUEI e o CHQ. O IRDI parte de concepções psicanalíticas, já o AUQUEI e o CHQ partem de concepções médicas de pesquisa.

Ao tentar correlacionar de alguma forma resultados obtidos com um instrumento baseado na perspectiva psicanalítica e outros dois instrumentos que provêm de uma perspectiva médica, estamos tentando ampliar o debate entre áreas de saber distintas.

O AUQUEI apresenta 26 questões ou domínios que exploram relações familiares, sociais, atividades, saúde, funções corporais e separação.

Operacionalmente, consiste em uma autoavaliação feita pela criança com o suporte de quatro imagens, que expressam diferentes estados emocionais, associados a diversos domínios da vida. Inicialmente, solicita-se à criança que ela apresente uma experiência própria vivida perante cada uma das alternativas reportadas pelas quatro imagens. Uma vez identificados os estados emocionais correspondentes, passa-se à leitura das questões, pedindo que ela assinale, sem tempo definido, a resposta que mais corresponde ao domínio proposto. Os escores podem variar de 0 a $3(0$ = muito infeliz; 1 = infeliz; 2 = feliz; 3 = muito feliz), e a escala possibilita a obtenção de um escore único, resultante da somatória dos escores atribuídos aos itens.
Segundo Assumpção Júnior, Kuczynski, Sprovieri e Aranha (2000, p. 121), "isso permite que a criança compreenda as situações e apresente sua própria experiência. A escala permite, assim, obter um perfil de satisfação da criança diante de diferentes situações".

Para as finalidades deste estudo, tem-se que a noção de qualidade de vida obtida com o AUQUEI é um indicador de saúde que permite mostrar o que a criança é capaz de elaborar sobre diferentes aspectos de sua vida, trazendo experiências próprias e se posicionando diante das questões que lhe são propostas.

A consideração da expressão da qualidade de vida não se esgota nessa concepção e, por isso, outro instrumento foi utilizado, o Child Health Questionnaire (CHQ-PF50) em sua versão destinada aos pais (parent form).

O CHQ é um instrumento de avaliação destinado a mensurar dois componentes fundamentais da saúde da criança: o bem-estar físico e o bem-estar psicossocial. É aplicável em crianças a partir de 5 anos de idade, possui 15 conceitos em saúde, cada um com pontuação de 0 a 100 . Maior pontuação indica melhor estado de saúde, de grau de satisfação e de bem-estar, resultando em dois índices sumários, o físico (PhS) e o psicossocial (PsS).

O CHQ já foi traduzido e validado em mais de 30 países por meio de uma pesquisa de adaptação transcultural que se mostrou válida na obtenção de instrumentos confiáveis e sensíveis às variações sociodemográfica e socioeconômica de cada país participante da pesquisa (Ruperto et al., 2001).

Podemos considerar que, para a finalidade deste estudo, o AUQUEI nos aproxima da definição de qualidade de vida do ponto de vista da criança, enquanto o CHQ nos possibilita ter uma noção da qualidade de vida relacionada ao estado de saúde do ponto de vista dos pais. Logo, justifica-se a escolha pela utilização destes instrumentos ao nos permitirem perspectivas distintas sobre a qualidade de vida da criança, além de sua ampla utilização na literatura sobre o tema e sua validação para aplicação no Brasil.

\section{Método}

Para verificar em que medida resultados referentes a condições psíquicas e de desenvolvimento obtidos com a aplicação do IRDI associam-se com resultados referentes à qualidade de vida da criança aos seis anos, foram utilizados dois instrumentos: o AUQUEI (preenchido a partir de entrevista com a criança) CHQ (preenchido a partir de entrevista com os pais) - ambos aplicados às cegas, isto é, sem o conhecimento prévio sobre os resultados dos participantes no IRDI.

Das 280 crianças que foram acompanhadas na pesquisa IRDI aos 3 anos, 114 pertenciam às instituições de saúde da cidade São Paulo, divididas da seguinte forma: 29 do HU-USP; 40 do Centro de Saúde Escola Samuel Barnsley Pessoa e 45 do Programa Einstein na Comunidade de Paraisópolis. 
Foi possível recuperar parte $(40,3 \%)$ da amostra original. Ao todo, foi realizado o acompanhamento de 46 crianças, distribuídas da seguinte maneira entre os centros: 14 crianças do Hospital Universitário da USP, 18 crianças do Centro de Saúde Escola Samuel Barnsley Pessoa e 14 crianças do Programa Einstein na Comunidade de Paraisópolis.

Para responder à pergunta que interroga sobre a associação entre resultados do IRDI e qualidade de vida foi utilizado um método estatístico. Os dados foram analisados pelo pacote estatístico SPSS versão 17.0 para Windows e pelo Microsoft Office Excel 2003. A análise foi realizada por meio de estatística descritiva, que consistiu em análise de tabelas de frequência, teste de Qui-quadrado e teste exato de Fisher para verificar a associação entre as variáveis. Em todos os testes realizados foi adotado o nível de significância de 5\%.

Os escores do IRDI foram classificados em caso e não caso da seguinte forma: crianças consideradas caso foram aquelas que apresentaram 2 ou mais indicadores ausentes e crianças consideradas não caso foram as que apresentaram um ou nenhum indicador ausente.

Os escores do AUQUEI variam de 0 a 3 e maiores escores significam melhor qualidade de vida. A escala possibilita a obtenção de um escore único, resultante da somatória dos escores atribuídos aos itens. Para efeito de discussão dos escores totais, utilizou-se a nota de corte 48 , obtida e proposta por Assumpção Júnior et al. (2000). Crianças que apresentaram escore abaixo de 48 foram consideradas com baixa qualidade de vida e crianças cujo escore foi igual ou superior a 48 foram consideradas com alta qualidade de vida.

Dos escores que se pode obter com o CHQ, foram utilizados os índices sumários referentes à condição física $(\mathrm{PhS})$ e à condição psicossocial $(\mathrm{PsS})$ da criança. $\mathrm{O}$ índice físico é composto pelos seguintes conceitos: função física $(\mathrm{PF})$, comportamento físico-social (RP), percepções sobre a saúde geral (GH), dores (BP), impacto no tempo dos pais (PT) e impacto emocional nos pais (PE). O índice psicossocial é composto pelos conceitos: impacto no tempo dos pais (PT), impacto emocional nos pais (PE), comportamento social (REB), autoestima (SE), saúde mental (MH) e comportamento (BE). Os conceitos têm variação de 1 a 5 e de 1 a 6, pontuados mediante o uso da escala de Likert descrito por Escobar (2005) e Landgraf, Abetz e Ware (1999). Os maiores valores correspondem a um melhor estado de saúde, com exceção dos conceitos $\mathrm{BP}, \mathrm{BE}, \mathrm{SE}, \mathrm{GH}, \mathrm{PE}$ e $\mathrm{MH}$, que foram recodificados. Os escores de cada conceito foram transformados para escala de 0 a 100 de acordo com a seguinte fórmula:

$$
\begin{gathered}
\text { Escore transformado }=(\text { Escore observado }- \\
\text { Menor escore possível })
\end{gathered}
$$

Range dos escores (Maior escore possível Menor escore possível)

Fonte: Landgraf et al. (1999)
Depois disso, foi calculado o Z-escore para cada um dos dez conceitos, utilizando como referência populacional a média e o desvio-padrão da população dos Estados Unidos. O Z-escore corresponde a cada escore transformado menos a média da população norte-americana referente a cada conceito dividido pelo desvio-padrão da mesma população, como na seguinte fórmula:

$$
\frac{\text { Z-escore }=\text { Escore transformado }- \text { Média }(E U A)}{\text { Desvio padrão }(E U A)}
$$

Fonte: Landgraf et al. (1999)

Após a padronização dos resultados em Z-escores, dois índices sumários foram gerados: o índice bruto de bem-estar físico (PhS) e o índice bruto de bem-estar psicossocial (PsS), obtidos por agregação dos Z-escores ponderados pelos coeficientes dos fatores físico e psicossocial sugeridos por Escobar (2005), Landgraf et al. (1999) e Ruperto et al. (2001), a partir dos estudos com a população geral dos Estados Unidos e seis amostras clínicas. As médias, os desvios-padrão e os coeficientes dos fatores físico e psicossocial utilizados como referência neste trabalho são os mesmos com que têm trabalhado pesquisadores brasileiros que se utilizam do CHQ-PF50.

Para efeito de comparação dos resultados obtidos nesta pesquisa com outras que utilizam o instrumento CHQ-PF50, as pontuações obtidas nessa etapa para os respectivos índices sumários brutos foram convertidas, a fim de que a distribuição dos dois índices gerados tenha média 50 e desvio padrão igual a 10, como indicado a seguir:

$$
\begin{aligned}
& \mathrm{PhS}=(\text { PhSraw } \times 10)+50 \\
& \mathrm{PsS}=(\text { PsSraw x 10)+50 }
\end{aligned}
$$

Valores acima de 50 indicam uma situação favorável de qualidade de vida, como aponta Escobar (2005). Logo, os índices PhS e PsS obtidos foram classificados em satisfatório se igual ou acima de 50 e insatisfatório se abaixo de 50.

Alguns autores têm utilizado a mediana dos escores obtidos nesse instrumento. Entretanto, para fins de comparação de resultados entre diferentes estudos, a média tem sido a medida de escolha, razão pela qual optamos por seu uso.

\section{Resultados}

A pesquisa mostrou que $93,5 \%$ dos entrevistados era o pai ou a mãe biológica da criança. Destes, $71,7 \%$ eram casados. Em relação ao grau de escolaridade, 34,8\% indicaram ser analfabetos ou ter até o $4^{\circ}$ ano do ensino fundamental, e $21,7 \%$ completaram curso superior. A maioria dos entrevistados $(73,9 \%)$ referiu renda mensal familiar 
maior que 1 salário mínimo per capita. Em relação à distribuição étnica referida na amostra, 56,5\% consideraram-se caucasianos, 39,1\% afrodescendentes e $4,3 \%$ orientais. A amostra foi composta por 46 crianças, sendo $26(56,5 \%)$ do sexo masculino e $20(43,5 \%)$ do sexo feminino. Do total, 20 $(43,5 \%)$ crianças alegaram ter concluído o jardim da infância, e $26(56,5 \%)$, o $2^{\circ}$ ano do ensino fundamental.

\section{Associação entre IRDI e AUQUEI}

Primeiramente, verificamos a associação entre resultados do IRDI e qualidade de vida a partir dos resultados obtidos no AUQUEI. Antes de apresentar os resultados obtidos a partir dessa associação, lembremos que das 46 crianças investigadas, $34(73,9 \%)$ foram não caso, e 12 (26,1\%), caso no IRDI. Quanto aos resultados obtidos no AUQUEI, as análises indicaram que $33(71,7 \%)$ crianças apresentaram alta qualidade de vida, e 13 (28,3\%), baixa qualidade de vida.

Os resultados da análise estatística apontaram que não há evidência de associação significativa entre o IRDI e a qualidade de vida relatada no AUQUEI $(p=0,717)$. Das 34 crianças consideradas não caso, 73,5\% apresentaram alta qualidade de vida e 26,5\%, baixa qualidade de vida. Das 12 crianças consideradas caso, 66,7\% apresentaram alta qualidade de vida e 33,3\%, baixa qualidade de vida. Como podemos observar na Tabela 1.

Tabela 1

\begin{tabular}{|c|c|c|c|c|}
\hline$I R D I$ & $\begin{array}{l}\text { Alta } \\
Q V\end{array}$ & $\begin{array}{c}\text { Baixa } \\
Q V\end{array}$ & Total & $\begin{array}{c}\text { Nivel de } \\
\text { significância } \\
\text { (p) }\end{array}$ \\
\hline Caso & $8(66,7 \%)$ & $\begin{array}{c}4 \\
(33,3 \%)\end{array}$ & $12(100 \%)$ & \multirow{2}{*}{0,717} \\
\hline Não caso & $\begin{array}{c}25 \\
(73,5 \%)\end{array}$ & $\begin{array}{c}9 \\
(26,5 \%)\end{array}$ & $34(100 \%)$ & \\
\hline
\end{tabular}

Fonte: Pesquisa de campo em Centros de Saúde de São Paulo (Di Paolo, 2010)

\section{Associação entre IRDI e CHQ}

Posteriormente, verificamos a associação entre resultados do IRDI e de qualidade de vida a partir dos resultados obtidos no CHQ. As análises indicaram que 28 (60,9\%) crianças apresentaram escore satisfatório para o índice físico (PhS), e 18 (39,1\%), insatisfatório para o mesmo fator; 27 (58,7\%) crianças apresentaram escore satisfatório para o índice psicossocial (PsS), e 19 (41,3\%), insatisfatório para o mesmo fator.

Das 34 crianças consideradas não caso, 58,8\% apresentaram índice satisfatório, e 41,2\%, insatisfatório para o bem-estar físico; em relação ao bem-estar psicossocial, 67,6\% das mesmas crianças apresentaram índice satisfatório, e 32,4\%, insatisfatório. Das 12 crianças consideradas caso, 66,7\% apresentaram índice satisfatório, e 33,3\%, insatisfatório para o bem-estar físico; em relação ao bem-estar psicossocial, 33,3\% das mesmas crianças apresentaram índice satisfatório, e $66,7 \%$, insatisfatório. Os resultados da análise estatística apontaram que o IRDI tem capacidade maior para predizer qualidade de vida relacionada ao índice psicossocial $(p=0,049)$ do que para predizer qualidade de vida relacionada ao índice físico $(\mathrm{p}=0,739)$, como podemos observar na Tabela 2.

Tabela 2

Percentual obtido a partir da associação entre IRDI e CHQ

\begin{tabular}{|c|c|c|c|c|}
\hline \multirow[t]{2}{*}{ IRDI } & \multicolumn{2}{|c|}{$P h S$} & \multirow[t]{2}{*}{ Total } & \multirow{2}{*}{$\begin{array}{c}\text { Nivel de } \\
\text { significância } \\
\text { (p) }\end{array}$} \\
\hline & Satisfatório & Insatisfatório & & \\
\hline Caso & $8(66,7 \%)$ & $4(33,3 \%)$ & $12(100 \%)$ & \\
\hline $\begin{array}{l}\text { Não } \\
\text { caso }\end{array}$ & $20(58,8 \%)$ & $14(41,2 \%)$ & $\begin{array}{c}34 \\
(100 \%)\end{array}$ & 0,739 \\
\hline \multirow{2}{*}{ IRDI } & \multicolumn{2}{|c|}{$P_{S S}$} & \multirow{2}{*}{ Total } & \multirow{2}{*}{$\begin{array}{c}\text { Nível de } \\
\text { significância } \\
\qquad(p)\end{array}$} \\
\hline & Satisfatório & Insatisfatório & & \\
\hline Caso & $4(33,3 \%)$ & $8(66,7 \%)$ & $12(100 \%)$ & \\
\hline $\begin{array}{l}\text { Não } \\
\text { caso }\end{array}$ & $23(67,6 \%)$ & $11(32,4 \%)$ & $\begin{array}{c}34 \\
(100 \%)\end{array}$ & 0,049 \\
\hline
\end{tabular}

Fonte: Pesquisa de campo em Centros de Saúde de São Paulo (Di Paolo, 2010)

Dos 31 indicadores, podemos destacar o 16 $(p=0,064)$ como aquele que se mostrou mais próximo do que consideramos estatisticamente significante. Como visto na Figura 1, o IRDI 16 é: "a criança demonstra gostar ou não de alguma coisa".

\section{Associação entre AUQUEI e CHQ}

Para fins de comparação com estudos que abordam a concordância da noção de qualidade de vida entre respostas de pais e filhos, realizamos uma associação entre os resultados obtidos no AUQUEI e no CHQ.

Ao observar a Tabela 3, é possível notar que das 33 crianças que apresentaram alta qualidade de vida no AUQUEI, 60,6\% apresentaram índice satisfatório, e 39,4\%, insatisfatório para o índice de bem-estar físico no CHQ; em relação ao índice de bem-estar psicossocial, 63,6\% das mesmas crianças apresentaram índice satisfatório, e 36,4\%, insatisfatório. Das 13 crianças que apresentaram baixa qualidade de vida no AUQUEI, 61,5\% apresentaram índice satisfatório, e 38,5\%, insatisfatório para o índice de bem-estar físico no $\mathrm{CHQ}$; em relação ao bem-estar psicossocial, 46,2\% das mesmas crianças apresentaram índice satisfatório, e 53,8\%, insatisfatório. 
Os resultados da análise estatística apontaram que a associação entre os resultados obtidos no AUQUEI e $\mathrm{o}$ índice psicossocial é mais significativa $(\mathrm{p}=0,331)$ do que a associação entre os resultados obtidos no AUQUEI e o índice físico $(p=1,000)$, como podemos observar na Tabela 3.

Tabela 3

Percentual obtido a partir da associação entre AUQUEI e CHQ

\begin{tabular}{|c|c|c|c|c|}
\hline \multirow[t]{2}{*}{ AUQUEI } & \multicolumn{2}{|c|}{$P h S$} & \multirow[t]{2}{*}{ Total } & \multirow{2}{*}{$\begin{array}{c}\text { Nivel de } \\
\text { significância } \\
(p)\end{array}$} \\
\hline & Satisfatório & Insatisfatório & & \\
\hline \multirow{2}{*}{$\begin{array}{c}\text { Alta } \\
\text { qualidade } \\
\text { de vida } \\
\text { Baixa } \\
\text { qualidade } \\
\text { de vida }\end{array}$} & $20(60,6 \%)$ & $13(39,4 \%)$ & $\begin{array}{c}33 \\
(100 \%)\end{array}$ & \multirow{2}{*}{1,000} \\
\hline & $8(61,5 \%)$ & $5(38,5 \%)$ & $\begin{array}{c}13 \\
(100 \%)\end{array}$ & \\
\hline \multirow{2}{*}{ AUQUEI } & \multicolumn{2}{|c|}{$P_{S S}$} & & \multirow{2}{*}{$\begin{array}{c}\text { Nivel de } \\
\text { significância } \\
(p)\end{array}$} \\
\hline & Satisfatório & Insatisfatório & Total & \\
\hline $\begin{array}{c}\text { Alta } \\
\text { qualidade } \\
\text { de vida }\end{array}$ & $21(63,6 \%)$ & $12(36,4 \%)$ & $\begin{array}{c}33 \\
(100 \%)\end{array}$ & \multirow{2}{*}{0,3} \\
\hline $\begin{array}{c}\text { Baixa } \\
\text { qualidade } \\
\text { de vida }\end{array}$ & $6(46,2 \%)$ & $7(53,8 \%)$ & $\begin{array}{c}13 \\
(100 \%)\end{array}$ & \\
\hline
\end{tabular}

Fonte: Pesquisa de campo em Centros de Saúde de São Paulo (Di Paolo, 2010)

Nota-se, descritivamente, que a maior parte das crianças consideradas com alta qualidade de vida no AUQUEI apresentou índices físico e psicossocial satisfatórios na avaliação do CHQ-PF50. No entanto, quando nos detemos na observação das colunas da tabela, vemos que a concentração de crianças com índices físico e psicossocial insatisfatórios do CHQ na situação de alta qualidade de vida da AUQUEI é maior do que sua concentração na situação de baixa qualidade de vida da AUQUEI. O que revela uma discordância significativa entre respostas aos dois instrumentos utilizados neste trabalho.

\section{Discussão}

Até hoje, numerosos instrumentos são utilizados com a finalidade de investigar a qualidade de vida, noção de difícil conceituação e mensuração. Neste estudo, partimos da definição da Organização Mundial da Saúde (OMS), que conceitua qualidade de vida como sendo a percepção do indivíduo de sua posição na vida no contexto cultural e no sistema de valores em que ele vive e em relação aos seus objetivos, expectativas, preocupações e desejos (The Whoqol Group, 1995).

Pode-se afirmar que o que se considera manifestação de qualidade de vida é tão diversificado como as suas formas de avaliação, não existindo consenso. Na literatura, notam-se inúmeras controvérsias teóricas e metodológicas, especialmente pelo caráter subjetivo da expressão do conceito e, ainda, pelos vários fatores que interferem na sua construção.

Em revisão de literatura sobre qualidade de vida em crianças e adolescentes com distrofia muscular de Duchenne (DMD) no Brasil, Melo, Valdés e Pinto (2005) detectaram dois instrumentos disponíveis para avaliação de qualidade de vida em crianças brasileiras: o AUQUEI e o CHQ, ambos já traduzidos para o português e adaptados para aplicação na população brasileira.

O AUQUEI foi desenvolvido por Manificat e Dazord (1997) em Lyon (França) com 95 crianças, das quais 60\% não apresentavam problemas de saúde, $26 \%$ eram crianças que haviam passado por transplante renal e $14 \%$ soropositivas para HIV, com idades entre 4 e 12 anos. O estudo apresentou propriedades psicométricas satisfatórias.

No Brasil, o instrumento foi traduzido e validado por Assumpção Júnior et al. (2000) em um estudo com 353 crianças saudáveis, com idades entre 4 e 12 anos, pertencentes a uma escola privada da zona sul de São Paulo, atestando suas propriedades psicométricas e obtendo uma nota de corte de 48 , abaixo da qual a qualidade de vida das crianças estudadas foi considerada prejudicada.

O CHQ-PF50 é um questionário desenvolvido para crianças com ou sem patologias crônicas. Foi elaborado e padronizado para uso na General Social Survey (GSS), uma pesquisa representativa sobre saúde da população, conduzida nos Estados Unidos entre 1994 e 1995. Os resultados obtidos têm sido tomados como base em discussões sobre avaliação de saúde e de qualidade de vida em crianças.

Sua tradução e validação para população brasileira foi realizada por Machado et al. (2001) em estudo realizado com pais de 471 crianças e adolescentes de ambos os sexos entre 6 e 18 anos, dos quais 157 eram portadores de artrite reumatoide juvenil (ARJ) e 314 eram saudáveis. Como conclusão, o estudo apontou excelentes propriedades psicométricas do instrumento, assim como sua confiabilidade e validade para a avaliação dos índices físico e psicossocial de crianças com ARJ.

Os resultados obtidos a partir da associação entre IRDI e AUQUEI coincidiram com estudos de qualidade de vida realizados com amostragem infantil que buscam uma correlação entre transtornos mentais e a noção de qualidade de vida na infância. Segundo os estudos de Elias e Assumpção Júnior (2006) e de Gottrand et al. (2005), não há diferença estatisticamente significativa entre a percepção de qualidade de vida de crianças que apresentam alguma patologia e a percepção de crianças consideradas saudáveis.

O IRDI apontou não ser capaz de prever qualidade de vida a partir do ponto de vista da criança. Pode-se conjecturar que detectar precocemente que a criança pode vir a desenvolver algum problema de desenvolvimento não influencia significativamente sua qualidade de vida. Assim, independentemente da quantidade de indicadores ausentes de 0 a 18 meses, a criança pode apresentar um alto ou baixo índice de qualidade de vida, o que dependeria de outras variáveis. Outra possibilidade de compreensão para este 
resultado é a de que a expressão da qualidade de vida considerada pelo AUQUEI não é sensível à condição psíquica da criança para a qual o IRDI foi estabelecido.

No entanto, quando relacionamos os resultados do IRDI às respostas dos pais sobre qualidade de vida chegamos a resultados diferentes dos observados no AUQUEI. A associação realizada entre o IRDI e o índice psicossocial do CHQ se mostrou estatisticamente significante, indicando que o IRDI apresenta maior capacidade para predizer qualidade de vida relacionada ao índice psicossocial $(\mathrm{p}=0,049)$ do que relacionada ao físico $(\mathrm{p}=0,739)$, como pudemos observar na Tabela 2. Os resultados indicaram que $66,7 \%$ das crianças consideradas caso no IRDI apresentaram índice psicossocial insatisfatório de qualidade de vida a partir do relato de seus pais. A expressão da qualidade de vida considerada pelo CHQ mostrou-se sensível à condição psíquica da criança para a qual o IRDI foi estabelecido.

Como apontam os estudos de Krab et al. (2009), de Speyer, Herbinet, Vuillemin, Chastagner e Briançon (2009) e de Upton, Lawford e Eiser (2008), diante de uma patologia orgânica já instalada na criança, os pais tendem a apontar índices de qualidade de vida mais baixos do que os relatados pelas crianças. Dado que, para os mesmos resultados de IRDI, crianças responderam ao AUQUEI tendendo a não associar risco (no IRDI) e qualidade de vida considerada insatisfatória, e pais responderam ao CHQ tendendo a fazer tal associação, podemos conjecturar que, além do constructo de cada instrumento, a diferença de sujeito experimental influencia a associação investigada, o que concorda com a literatura internacional sobre $\mathrm{o}$ assunto.

Podemos considerar que, se na presente pesquisa os pais apontaram qualidade de vida mais baixa para crianças que foram caso no IRDI, as mesmas podem apresentar atualmente traços psicopatológicos. É digno de nota que o IRDI tem como foco o papel do enlaçamento com o outro, o que se faz fundamental no processo de constituição subjetiva da criança e também em seu desenvolvimento, assim, o escore do CHQ em que as crianças foram avaliadas significativamente com qualidade de vida insatisfatória foi o índice psicossocial, contendo aspectos que se referem aos laços estabelecidos pelas crianças.

Assim, podemos considerar que o IRDI é capaz de detectar uma tendência de ocorrência de problemas expressa nos 18 primeiros meses de vida que se estende ao longo do desenvolvimento da criança pelo menos até o sexto ano de vida, levando ao impacto em sua qualidade de vida a partir da perspectiva de seus pais.

Uma questão metodológica que se impõe a partir desses resultados é a de que a noção de qualidade de vida com que um instrumento opera pode ser um fator determinante na medição do índice do que é considerada alta e baixa qualidade de vida para o sujeito. Neste estudo, uma das perspectivas que fundamenta tal definição foi concebida a partir da pesquisa realizada por Assumpção Júnior et al. (2000) com 353 crianças saudáveis, o que nos permite questionar se é válido utilizar as mesmas questões em uma população saudável e em uma população que sofre de alguma patologia.

Os resultados obtidos até o momento podem mostrar que crianças que sofrem de alguma patologia podem viver de forma satisfatória na sua condição ou, alternativamente, que não foi possível avaliar sua qualidade de vida a partir da noção que sustenta o instrumento utilizado. Há que se indagar, portanto, se a noção de qualidade de vida com a qual a literatura vem trabalhando é sensível ao sofrimento de portadores de psicopatologias. Se mudarmos a noção de qualidade de vida, encontraremos diferenças significativas entre portadores de psicopatologias e não portadores? Eis uma questão que precisaria de estudos mais amplos para possíveis verificações.

Além disso, vale lembrar que o IRDI é um instrumento psicanalítico embasado fortemente na noção de inconsciente. É possível afirmarmos que o sofrimento apontado pela perspectiva psicanalítica, dada sua fundamentação na noção de inconsciente, pode não ser detectado por uma noção de qualidade de vida apoiada na percepção, atributo consciente, ou pode não ser detectado nos aspectos elencados para serem percebidos pelos sujeitos da pesquisa. Essa é uma questão metodológica fundamental se quisermos utilizar um instrumento para avaliação de qualidade de vida em saúde pública, como tem sido a tendência notada na literatura internacional.

A tendência notadamente observada nos estudos mais recentes aponta para uma relação não significativa entre condição psíquica sintomática e qualidade de vida, como se a primeira não tivesse influência significativa sobre a segunda. Contudo, o que observamos a partir do uso do AUQUEI neste trabalho é que tal instrumento mostrouse problemático dos pontos de vista teórico e metodológico. A respeito do primeiro ponto de vista, temos questões que indagam se a criança está ou não está "feliz" nas situações propostas no questionário. Antes disso, é necessário definir "felicidade" para tomá-la como elemento de referência de qualidade de vida.

Em relação ao outro ponto de vista, o metodológico, temos um instrumento que busca medir a percepção subjetiva da qualidade de vida da criança a partir de suas sensações de "felicidade" e de "infelicidade", o que pode ser facilmente confundido com satisfação ou alívio, por exemplo, por uma criança angustiada em separar-se da mãe, condição que pode chegar a ser incapacitante e que, portanto, pode levar à noção de baixa qualidade de vida, fazendo uma referência de extrema felicidade ao ficar colada na mãe. São tão clássicos como surpreendentes os casos de sequestrados que, em quadros de síndrome de Estocolmo, dir-se-iam felizes por continuar com seus sequestradores, em evidente negação defensiva da violência a que foram submetidos. Decorre então que a noção de qualidade de vida é um conceito que não deveria ser reduzido a medidas de felicidade. Daí situarmos este instrumento como problemático e de difícil mensuração para o que se propõe.

Uma questão que se impõe a partir do estudo realizado é a necessidade de ampliar a noção de qualidade de 
vida com a qual a literatura vem trabalhando, no que diz respeito a sua sensibilidade diferencial para psicopatologia. Os resultados permitem interrogar, ainda, se os sujeitos portadores de alguma psicopatologia na infância são avaliados positivamente para qualidade de vida, porque, na sua percepção, a patologia não se constitui como um entrave à sua qualidade de vida, ou porque simplesmente não possuem as mesmas habilidades psíquicas de distinguir situações felizes e infelizes de seu cotidiano, isto é, não respondem da mesma maneira que uma criança com desenvolvimento saudável.

Além disso, trata-se de um instrumento que não está afinado teórica e metodologicamente ao IRDI, construído a partir do referencial teórico da Psicanálise. A proposta de estabelecer associações entre instrumentos de bases epistemológicas, semiológicas e metodológicas - e, ainda, que orientam formas de intervenção - distintas foi válida porque fomenta o debate entre áreas distintas do saber.

Em relação aos resultados observados na Tabela 3, pudemos notar que há maior associação entre AUQUEI e índice psicossocial (PsS) do CHQ ( $\mathrm{p}=0,331)$ do que entre AUQUEI e índice físico (PhS) do CHQ ( $\mathrm{p}=1,000)$. Tais dados nos levam à afirmação de que os fatores abordados no AUQUEI relacionados a condições de saúde da criança estão mais próximos dos fatores abordados no índice psicossocial do CHQ: impacto no tempo dos pais (PT), impacto emocional nos pais (PE), comportamento social (REB), autoestima
(SE), saúde mental (MH) e comportamento (BE). Fatores relacionados à condição de saúde física não se mostraram presentes de forma significativa no instrumento AUQUEI, o que pode ser um indício explicativo para este resultado.

Os estudos de Speyer et al. (2009) e de Upton et al. (2008) apontam que há uma tendência dos pais a superestimar o índice de qualidade de vida dos filhos quando estes se encontram saudáveis e a subestimá-lo quando estes se encontram em situação de enfermidade. Dessa forma, podemos atribuir essa discrepância observada entre os resultados do AUQUEI e do CHQ ou ao possível exagero da referência dos pais acerca da avaliação da qualidade de vida de seus filhos ou, então, a uma dificuldade do próprio instrumento AUQUEI em registrar problemas de qualidade de vida decorrentes de condições de saúde relacionadas ao bem-estar físico, por exemplo, como abordado no CHQ. Nota-se que o AUQUEI não aborda aspectos relacionados ao estado de saúde da criança tais como abordados pelo $\mathrm{CHQ}$, o que torna seu uso questionável, considerando a importância de tais aspectos na avaliação da qualidade de vida observada na literatura.

A análise estatística apontou manifestações de tendências do IRDI quanto à avaliação da qualidade de vida da criança, especialmente no que se refere aos aspectos psicossociais obtidos com o uso do CHQ-PF50. Pode-se destacar, assim, que o IRDI apresenta capacidade de predição para condições sintomáticas posteriores que podem afetar negativamente a qualidade de vida da criança.

\section{Association between signs of psychological distress up to 18 months and decrease in quality of life at six years of age}

Abstract: At the request of the Ministry of Health, a protocol of Clinical Risk Indicators for Child Development (IRDI) capable of detecting risk for psychic development disorders in infants aged between 0 and 18 months was developed and validated. This study aimed to verify the degree of association between IRDI and two other instruments for assessing quality of life: Autoquestionnaire Qualité de Vie Enfant Image - AUQUEI and Child Health Questionnaire - CHQ, both already validated in Brazil and applied at the age of six years. The sample comprised 46 children from three health centers in São Paulo, Brazil. The results indicated that IRDI was not sensitive to the assessment of quality of life achieved by using AUQUEI. However, a statistically significant association found between the results of IRDI and CHQ showed higher capacity of IRDI to predict quality of life related to psychosocial index than the physical index of the same instrument.

Keywords: quality of life, child development, psychoanalysis.

\section{Association entre des signaux de détresse psychologique jusqu'à dix-huit mois et qualité de vie inférieure à six ans}

Résumé: À la demande du Ministère de la Santé, un groupe de psychanalystes a développé et validé un protocole d'Indicateurs de Risque Cliniques pour le Développement de l'Enfant (IRDI), capable de détecter les risques de troubles dans le développement mentaux chez bébés d'âge entre 0 et 18 mois. Cette étude visait à déterminer le degré d'association entre l'IRDI et des deux autres instruments qui évaluent la qualité de vie : I'Autoquestionnaire Qualité de Vie Enfant Imagé (AUQUEI) et le Child Health Qtuestionnaire (CHQ). Les deux ont été validés au Brésil et on été appliqués aux enfants à l'áge de six ans. L'échantillon comprenait 46 enfants hospitalisés dans trois centres de santé à São Paulo, au Brésil. Les résultats indiquent que l'IRDI n'est pas sensible à l'évaluation de la qualité de vie obtenue par I'AUQUEl. Cependant, l'association statistiquement significatif trouvée dans les résultats a montrée une capacité du IRDI de prédire la qualité de vie liée à l'index psychosocial plus grande que de laquelle liée à l'index physique du même instrument.

Mots-clés: qualité de vie, développement de l'enfant, psychanalyse. 


\section{Asociación entre las señales del sufrimiento psíquico hasta los dieciocho meses y la disminución de la calidad de vida en los seis años de edad}

Resumen: Un protocolo de Indicadores clínicos de Riesgo para el Desarrollo Infantil (IRDI) fue desarrollado y validado a demanda del Ministerio de Salud para detectar los riesgos de trastornos psíquicos de desarrollo en los niños de 0 a 18 meses. Este estudio tuvo como objetivo determinar el grado de asociación entre el IRDI y otros dos instrumentos que evalúan la calidad de vida: el Autoquestionnaire Qualité de Vie Enfant Imagen (AUQUEI) y el Child Health Questionnaire (CHQ), ambos ya validados en Brasil y aplicados a los seis años de edad. La muestra consta de 46 niños de tres centros de salud de São Paulo. Los resultados indicaron que el IRDI no se mostró sensible a la evaluación de la calidad de vida obtenida mediante el uso del AUQUEI. Sin embargo, la asociación estadísticamente significativa encontrada entre los resultados obtenidos en el IRDI y CHQ mostró una mayor capacidad del IRDI para predecir la calidad de vida relacionada al índice psicosocial que al físico del mismo instrumento.

Palabras clave: calidad de vida, desarrollo infantil, psicoanálisis.

\section{Referências}

Assumpção Júnior, F. B., Kuczynski, E., Sprovieri, M. H., \& Aranha, E. M. G. (2000). Escala de avaliação de qualidade de vida (AUQUEI - AUTOQUESTIONNAIRE QUALITÉ DE VIE ENFANT IMAGÉ) Validade e confiabilidade de uma escala para qualidade de vida em crianças de 4 a 12 anos. Arquivos de Neuropsiquiatria, 58(1), 119-127.

Brasil. Ministério da Saúde. (2007). Caderneta de saúde da criança. Recuperado de http://portal.saude.gov.br/portal/ arquivos/pdf/caderneta_crianca_2007_29.pdf.

Di Paolo, A. F. (2010). Estudo exploratório dos Indicadores Clínicos de Risco para o Desenvolvimento Infantil e da Avaliação Psicanalítica aos 3 anos para avaliar qualidade de vida e condição sintomática aos seis anos (Dissertação de Mestrado). Instituto de Psicologia, Universidade de São Paulo.

Elias, A. V., \& Assumpção Júnior, F. B. (2006). Qualidade de vida e autismo. Arquivos de Neuropsiquiatria, 64(2-A), 295-299.

Escobar, A. M. U. (2005). Estudo das condições de saúde das crianças de 5 a 9 anos de idade residentes no Distrito do Butantã na cidade de São Paulo (Tese de Livre-docência). Faculdade de Medicina, Universidade de São Paulo.

Gottrand, F., Staszewski, P., Colomb, V., Loras-Duclaux, I., Guimber, D., Marinier, E., et al. (2005). Satisfaction in different life domains in children receiving home parenteral nutrition and their families. Journal of Pediatrics, 146(6), 793-797.

Krab, L. C., Oostenbrink, R., de Goede-Bolder, A., Aarsen, F. K., Elgersma, Y., \& Moll, H. A. (2009). Health-related quality of life in children with neurofibromatosis type 1: contribution of demographic factors, disease-related factors and behavior. Journal of Pediatrics, (154)3, 420-425.

Kupfer, M. C. M., Bernardino, L. M. F., Jerusalinsky, A. F., Rocha, P. S. B., Lerner, R., \& Pesaro, M. E. (2008). A pesquisa IRDI: resultados finais. In R. Lerner \& M. C. M. Kupfer (Orgs.), Psicanálise com crianças: clínica e pesquisa (pp. 221-230). São Paulo: Escuta.

Kupfer, M. C. M., Jerusalinsky, A. F., Bernardino, L. M. F., Wanderley, D., Rocha, P. S. B., Molina, S. E., et al. (2009). Valor preditivo de indicadores clínicos de risco para o desenvolvimento infantil: um estudo a partir da teoria psicanalítica. Latin American Journal of Fundamental Psychopathology Online, 6(1), 48-68.

Kupfer, M. C. M., Jerusalinsky, A. F., Bernardino, L. M. F., Wanderley, D., Rocha, P. S. B., Molina, S. E., et al. (2010). Predictive value of clinical risk indicators in child development: final results of a study based on psychoanalytic theory. Revista Latinoamericana de Psicopatologia Fundamental, 13(1), 16-30.

Landgraf, J. M., Abetz, L., \& Ware, J. E. (1999). Child health questionnaire (CHQ): a user's manual. Boston: HealthAct.

Lerner, R. (2011). Indicadores clínicos de risco para $o$ desenvolvimento infantil - IRDI: verificação da capacidade discriminativa entre autismo, retardo mental e normalidade (Tese de Livre-docência). Instituto de Psicologia, Universidade de São Paulo.

Lerner, R., \& Kupfer, M. C. M. (Orgs.). (2008). Psicanálise com crianças: clínica e pesquisa. São Paulo: Escuta.

Machado, C. S. M., Ruperto, N., Silva, C. H., Ferriani, V. P., Roscoe, I., Campos, L. M., et al. (2001). The Brazilian version of the Childhood Health Assessment Questionnaire (CHAQ) and the Child Health Questionnaire (CHQ). Clinical and Experimental Rheumatology, 19(1), 25-29.

Manificat, S., \& Dazord, A. (1997). Évaluation de la qualité de vie de l'enfant: validation d'un questionnaire, premiers résultats. Neuropsychiatrie de l'enfance et de l'adolescence, 45(3), 106-114.

Melo, E. L. A., Valdés, M. T. M., \& Pinto, J. M. S. (2005). Qualidade de vida de crianças e adolescentes com Distrofia Muscular de Duchenne. Revista de Pediatria, 27(1), 28-37.

Pesaro, M. E. (2010). Alcance e limites teórico-metodológicos da pesquisa multicêntrica de indicadores clínicos de risco para o desenvolvimento infantil (Tese de Doutorado). Instituto de Psicologia, Universidade de São Paulo.

Ruperto, N., Ravelli, A., Pistorio, A., Malattia, C., Cavuto, S., Gado-West, L., et al. (2001). Cross-cultural adaptation and psychometric evaluation of the Childhood Health 
Assessment Questionnaire (CHAQ) and the Child Health Questionnaire (CHQ) in 32 countries. Review of the general methodology. Clinical and Experimental Rheumatology, 19(23), 1-9.

Speyer, E., Herbinet, A., Vuillemin, A., Chastagner, P., \& Briançon, S. (2009). Agreement between children with cancer and their parents in reporting the child's healthrelated quality of life during a stay at the hospital and at home. Child Care Health and Development, 35(4), 489-495.

The Whoqol Group. (1995). The world health organization quality of life assessment (WHOQOL): position paper from the world health organization. Social Science and Medicine, 41(10), 1403-1409.

Upton, P., Lawford, J., \& Eiser, C. (2008). Parent-child agreement across child health-related quality of life instruments: a review of the literature. Quality of Life Research, 17(6), 895-913.
Recebido: 20/11/2013

Revisado: 17/05/2014

Aceito: 04/07/2014 